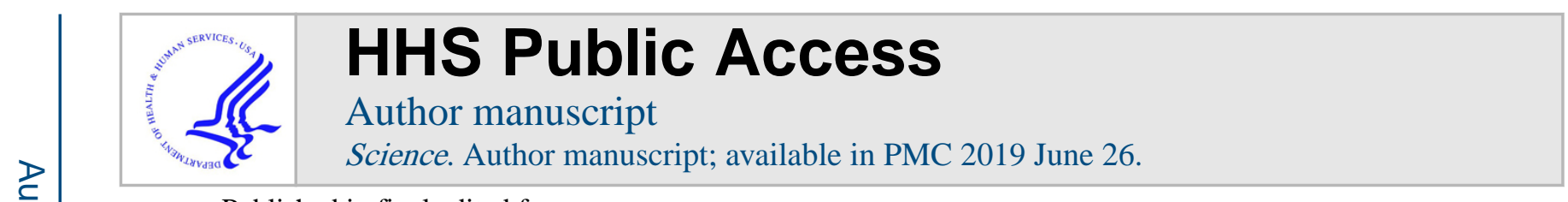

Published in final edited form as:

Science. 2008 August 15; 321(5891): 922-923. doi:10.1126/science.1162910.

\title{
Secret Weapon
}

\author{
Ryland F. Young III \\ Department of Biochemistry and Biophysics, Texas A\&M University, College Station, TX 77843- \\ 2128, USA.
}

The battle between bacteria and their viruses (bacteriophages) is, quantitatively, the dominant predator-prey relation in the biosphere, with an estimated $10^{30}$ infections per day. It is an unequal contest in many ways. Phages replicate prodigiously. Within 2 hours of the addition of a single $\mathrm{T} 7$ bacteriophage particle to a culture of 10 billion Escherichia coli cells, more than $99.9 \%$ of the bacteria are destroyed and 10 trillion virus particles are generated. And although, in an evolutionary sense, bacteria can "run" by generating receptor mutations that prevent phage binding, they cannot "hide"- phage mutate at such high frequency that every mutational evasion tried by bacteria is soon overcome by phages with altered specificity (1). Moreover, phages undergo unparalleled degrees of genomic recombination, so new specificities and other virulence features can spread rapidly to brethren and even unrelated phages. On page 960 in this issue, Brouns et al. (2) characterize a new kind of bacterial defense based on small RNA molecules that match short sequences in the phage DNA.

After binding to a receptor, a phage injects its genetic material into the bacterium, whereupon viral DNA and proteins are synthesized and new virions are assembled. A system called restriction-modification is a well-known bacterial defense against phage infection, in which a bacterium chemically modifies its own DNA at every occurrence of a particular restriction site, usually a short palin-dromic sequence. Wherever the sequence occurs in the newly injected phage DNA, it will not be modified and consequently, it will be cleaved by a corresponding restriction endonuclease, stopping the virus in its tracks. But occasionally the system fails, and if the DNA of even one virus becomes modified, all of its subsequent progeny will encounter no barrier in further infection cycles. Moreover, some phages use altered DNA bases that confound restriction enzymes, whereas others inject proteins that inhibit the restriction enzymes. Even more cleverly, phages may use a two-step injection process, in which only a small portion of phage DNA first enters the bacterium. This DNA encodes proteins that antagonize restriction enzymes. Cells also have suicide defenses, in which infections by certain phages leads to premature cell death, squelching the infection cycle before virus particles assemble.

Recently, a new kind of phage defense was discovered, based on loci called clusters of regularly interspaced short palindromic repeats (CRISPR) that are widespread in the DNA of bacteria and archaea (3-5). CRISPR loci consist of multiple short nucleotide repeats separated by unique spacer sequences and flanked by a characteristic set of CRISPR-

ryland@tamu.edu. 
associated (cas) genes (see the figure) $(6,7)$. The discovery that the spacers were often identical to short sequences in phage DNA, and that they seem to be constantly changing in bacteria, suggested that they were a kind of "memory of past genetic aggressions" (8) and might underlie some kind of defense against foreign DNA (9-11). This was confirmed in an elegant study by Barrangou et al. (3), in which a culture of Streptococcus thermophilus was challenged with phage. Rare phage-resistant bacteria were isolated that had acquired at least one new CRISPR spacer identical to a sequence in the phage DNA. By replacing the entire CRISPR repeat array in $S$. thermophilus with the new CRISPR spacers, phage resistance was conferred. Moreover, phage overcame resistant bacteria by mutating just 1 base pair within the sequence corresponding to the new spacer.

More recently, metagenomic analysis of archaea has indicated that CRISPR loci are extremely dynamic, with sequence changes occurring on a time scale of months, and that new spacers appear corresponding to phages coexisting in archaeal communities (biofilms) (12). An interesting twist from this analysis was that, at least for the phages in these biofilms, overcoming the CRISPR defense appeared to be primarily a matter of intense recombinational shuffling down to a scale of the size of CRISPR spacers, rather than mutations.

Although these genetic and genomic analyses $(3,12)$ clearly showed that the CRISPR defense is a fundamental aspect of bacterial and archaeal evolution, mechanistic insight was completely lacking. In a major step forward, Brouns et al. have reconstituted the CRISPR phenomenon in laboratory strains of $E$. coli, which, although it has CRISPR sequences, had not been shown to use the CRISPR defense against any known phages. The authors identify a multiprotein complex called CRISPR-associated complex for antiviral defense (Cascade), consisting of five Cas proteins (CasA to CasE), and show that it processes a long primary CRISPR transcript to 57-nucleotide fragments, each containing a unique spacer and bits of the flanking repeat sequences (see the figure). By cloning all five cas genes in different combinations into an E. coli strain lacking CRISPR sequences entirely, Brouns et al. show that only CasE is required for cleavage of the primary CRISPR transcript. Cascade processed primary CRISPR RNA, but not CRISPR RNA (with a different CRISPR repeat) from a different $E$. coli species, and could be copurified with the 57-nucleotide RNAs, indicating the formation of a ribonucleoprotein complex. Importantly, the authors could also construct a CRISPR defense against the bacteriophage lambda by engineering new spacers into the $E$. coli CRISPR locus, chosen from sites throughout the lambda genome. The artificial CRISPR array was efficient, reducing the ability of phage lambda to grow by a factor of 10,000,000. This effect depended on the presence of functional Cascade and expression of the cas 3 gene. In each case, the spacer sequences could be chosen from either the template or noncoding DNA strand of the phage genes, suggesting that the target of CRISPR is the phage DNA. This seems to be fundamentally different from the small inhibitory RNA strategy of eukaryotes, which suppresses viral gene expression by destroying corresponding messenger RNA.

The work by Brouns et al. has put at least the active defense aspect of the CRISPR system on track for thorough mechanistic and structural analysis. The ability to genetically and biochemically manipulate $E$. coli is far superior to other biological systems, and 
bacteriophage lambda is arguably the only biological entity for which we have nearly predictive understanding. It can be expected that rapid advances will be made in elucidating the molecular details for CRISPR gene expression, RNA processing, and the attack on the target phage.

Still obscure is how a bacterium acquires new spacer sequences. No one has reported a system for achieving this naturally, at efficiencies conducive to biochemical analysis. Moreover, we should anticipate that phages have developed clever evasions of the CRISPR system just as they have done for restriction enzymes. Considering that the origin of modern molecular biology is grounded in the study of bacteriophage, it is puzzling that this particular weapon in the phage-bacteria war remained a secret for so long.

\section{References}

1. Qimron U, Marintcheva B, Tabor S, Richardson CC, Proc. Natl. Acad. Sci. U.S.A 103, 19039 (2006). [PubMed: 17135349]

2. Brouns SJJ et al., Science 321, 960 (2008). [PubMed: 18703739]

3. Barrangou R et al., Science 315, 1709 (2007). [PubMed: 17379808]

4. Deveau H et al., J. Bacteriol 190, 1390 (2008). [PubMed: 18065545]

5. Horvath P et al., J. Bacteriol 190, 1401 (2008). [PubMed: 18065539]

6. Jansen R, Embden JD, Gaastra W, Schouls LM, Mol. Microbiol 43, 1565 (2002). [PubMed: 11952905]

7. Haft DH, Selengut J, Mongodin EF, Nelson KE, PLoS Comput. Biol 1, e60 (2005). [PubMed: 16292354]

8. Pourcel C, Salvignol G, Vergnaud G, Microbiology 151, 653 (2005). [PubMed: 15758212]

9. Bolotin A, Quinquis B, Sorokin A, Ehrlich SD, Microbiology 151, 2551 (2005). [PubMed: 16079334]

10. Mojica FJ, Diez-Villasenor C, Garcia-Martinez J, Soria E, J. Mol. Evol 60, 174 (2005). [PubMed: 15791728]

11. Makarova KS, Grishin NV, Shabalina SA, Wolf YI, Koonin EV, Biol. Direct 1, 7 (2006). [PubMed: 16545108]

12. Andersson AF, Banfield JF, Science 320, 1047 (2008). [PubMed: 18497291] 


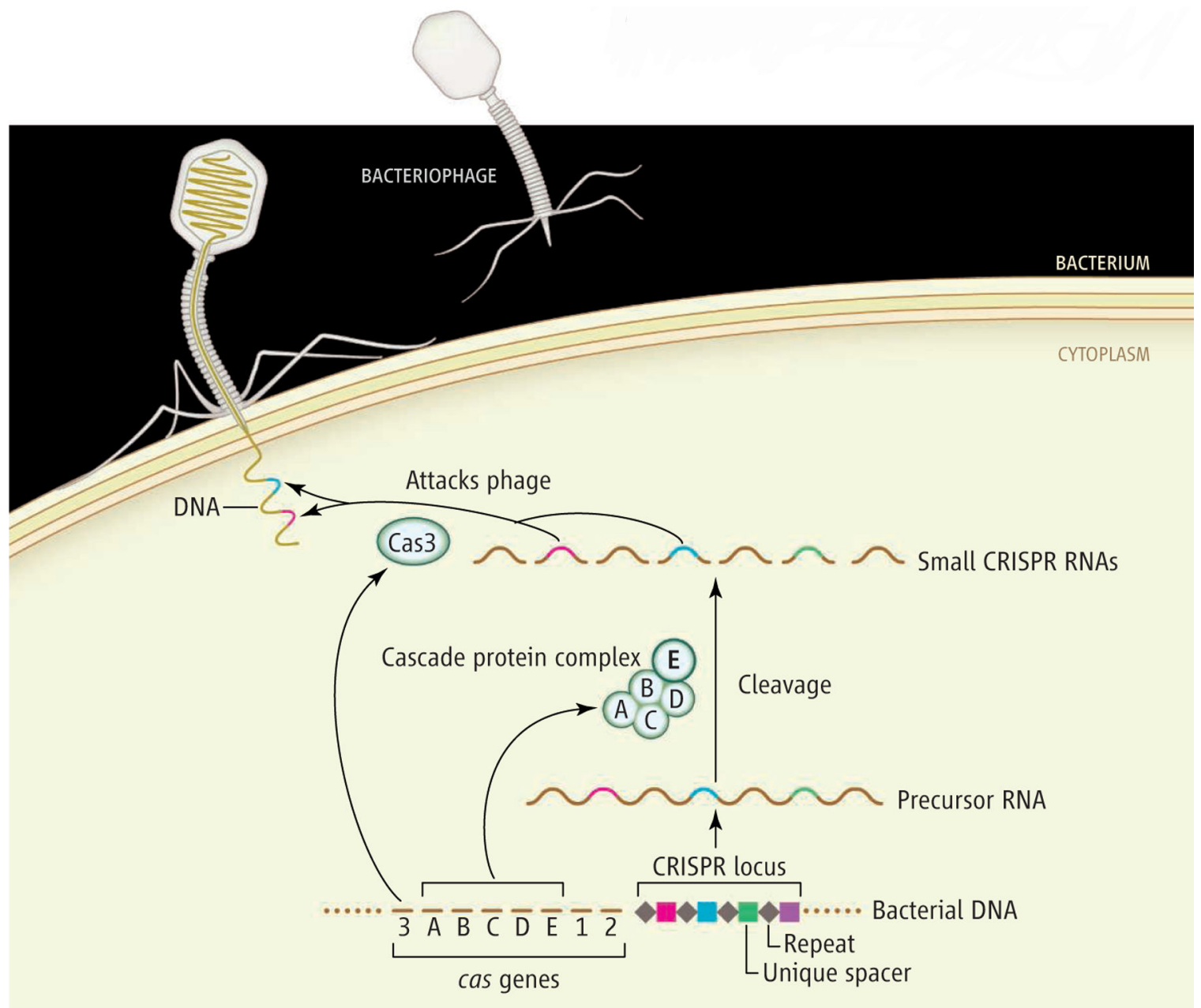

Figure. A CRISPR defense.

The CRISPR locus in $E$. coli is transcribed into a large precursor RNA, which is processed by the Cascade protein complex into short fragments that contain unique spacers identical to sequences in the phage DNA. Assisted by the protein Cas3, these small CRISPR RNAs block the phage infection cycle. 